\title{
A Finite Element Analysis of the Dynamic Response of a Thick Uniform Elastic Circular Plate Subjected to an Exponential Blast Loading
}

\author{
Aiyesimi Y. M ${ }^{1}$, A. A. Mohammed ${ }^{1, *}$, S. Sadiku ${ }^{2}$ \\ ${ }^{1}$ Department of Mathematics and Statistics, Federal University of Technology, Minna, Nigeria \\ ${ }^{2}$ Department of Civil Engineering, Federal University of Technology, Minna, Nigeria
}

\begin{abstract}
The result of the study of dynamic response of an elastic circular plate to blast load is presented in this research work. Finite element method is used to derive the equation of motion for the circular plate element under the influence of exponential impulse forces. System stiffness and mass matrices were drive. The effects of transverse shear deformation and rotatory inertia were included. From the numerically simulated results it is observed that the amplitude dies out quickly due to the effect of damping. The pulse duration $t_{d}$ is also one of the most important parameter because it gives serious influence to the vibration amplitude. It gives rise to the vibration amplitude on any small decrease on the pulse duration. It is also observed that the exponential blast loading brings faster rate of amplitude decay than those of triangular and sinusoidal blast loading.
\end{abstract}

Keywords Circular Plate, Finite Element, Blast Loading

\section{Introduction}

Structures may experience blast loads due to military actions, accidental explosions or terrorist activities. Such loads may cause severe damage or collapse due to their high intensity, dynamic nature, and usually different direction compared to common design loads. The occurrence of plate structure is extensive in various engineering constructions. Nonlinear vibration occurs very often in many applications of panels, particularly in aircraft. Aircraft skin - panels especially those near the exhaust of jet engines, are often subject to high levels of acoustic pressure. This may result in large amplitude, nonlinear vibration of panels. Extensive literature reviews on the nonlinear vibration of plates subjected to different loading are given by the following researchers. Rao et al (1993) presented a finite element method for the large amplitude free flexural vibration of unstifened and stiffened plates. For the simply supported square plate, non-linear frequency ratios from six different approaches were reported. The frequency ratio at $\omega_{\max } / h=1.0$ varied from a low of 1.2967 to a high of 1.5314. Chiang et al. (1991) developed a rectangular element with 24 degrees of freedom (6 DOF per node). The linearized updated-mode was used to study the harmonic forced vibration. Although the in-plane displacements were included in the formulation, only bilinear functions were employed for the in-plane displacement shape functions. Rao, S. R. A. et al (1992) use finite element

* Corresponding author:

adamualhaj@yahoo.com (A. A. Mohammed)

Published online at http://journal.sapub.org/ajcam

Copyright (C) 2011 Scientific \& Academic Publishing. All Rights Reserved method to study large - amplitude free flexural vibration of stiffened and unstiffened plates. They employed the total Lagrangian coordinates system to formulate the dynamic version of Von Karman's field equations where the stiffener has been elegantly modeled so that it may lie anywhere within the plate element. Chorng-Fuh L. and Ge-Tzung C. (1995) present a simple finite element analysis of axisymmetric vibration of annular and circular plates. They based the formulation of the finite element on elasticity theory which has no assumptions as in the conventional plate theory-based analysis. They show that different simply supported conditions can make significant differences in the prediction of axisymmetric vibration frequencies for both thin and thick plates. Ding H. et al (1999) experimentally studied free axisymmetric vibration of transversely isotropic piezoelectric circular plates. They used Finite Hankel transforms and the boundary variables in free terms are replaced for two kinds of boundary conditions to obtain ordinary differential equations with constant coefficients to derive axisymmetric state space for mulation of piezoelectric laminated circular plates. They found that exact solutions can be obtained for two kinds of boundary conditions and the solutions can be used to examine the validity of various plate theories and numerical calculation software. Aiyesimi, Y. M. (1999) investigates the dynamic analysis of an elastic plate with point-moving load. He determine Mathematically the vibrating conditions that give rise to the occurrence of natural frequency and the moving load constitute an external vibration. Aiyesimi, Y. M. (2000) studied the analytical investigation of the effect of amplitude ratio on the dynamic response of a viscoelastic plate subject to a cyclic moving load. The vibrating plate is incorporated with a damping 
foundation regarded as a viscoelastic foundation where he analyzed the effect of the amplitude ratio on the magnitude of the vibration and observed that the amplitude of vibration increases with the amplitude ratio. Amabili, M. et al (2003) experimentally studied large amplitude vibrations of a stainless - steel circular cylindrical panel supported at four edges. The nonlinear response to harmonic excitation of different magnitudes in the neighborhood of three resonances was investigated. Experiments showed that the curved panel exhibited a relatively strong geometric nonlinearly of softening type. Sathyamoorthy, M. (1996) studied the influence of transverse shear and rotatory inertia on non-linear vibration of circular plates. Effects of transverse shear deformation and rotatory inertia have been included in the Berger - type governing equations. He found that the present Berger - type formulation in the polar coordinate system is found to yield reasonable good results for plates with immovable boundary conditions. Ming-Hui, L. and Tsung-Chien, C. (2008) used inverse method to investigate blast load input estimation of the medium girder bridge. An adaptive weighted input estimation inverse methodology is applied to estimate the unknown time-varying blast loads in a truss structural system. The FEM is adopted to construct the state equation of the truss structure, and the Kalman filter is further combined with the adaptive weighted recursive least square estimator, which recursively estimates the unknown blast loads under a situation that the system involves the measurement and modeling errors. Their results indicate that the presented technique will have higher estimation ability adopting the precise measurement instrument. Van der Meer, L. J (2008) experiment the dynamic response of high-rise building structures to blast loading. The SDOF response is determined analytically for linear-elastic material behavior, idealized blast loads and without damping. The response is divided in three regimes: impulsive, dynamic and quasi-static. He concluded that the SDOF system is not conservative for response in the impulsive regime, whereas the quasi-static response is similar for both models. BLEVE (boiling liquid expanding vapor explosion) blast loading on high-rise buildings is likely in the impulsive regime. Olarewaju, A. J. et al (2011) Simulated and Verified the blast load duration for studying the response of underground horizontal and vertical pipes using finite element method. They used time integration technique in Abaqus/Explicit to solve the equation of motion. From their result of the response of underground pipes due to surface blast, for various periods considered, it was observed that duration of blast play a significant role in the response of pipes buried, most especially pipes buried in dense sand and undrained clay.

\section{Exponential Blast}

An actual bomb explosion, like most blast loads, has a random pressure - time history. However, such an explosion invariably has a sharp pressure rise followed by a time of decay. The pulse shapes shown in figure 1 . is the exponential blast wave

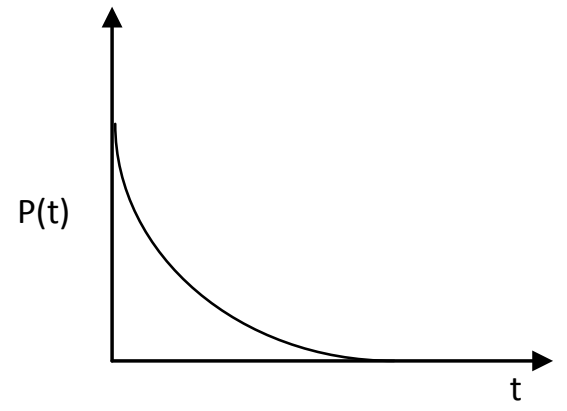

Figure 1. Exponential decay

The modified Friedlander exponential decay equation.

$$
p(t)=p_{m}\left(1-\frac{\mathrm{t}}{\mathrm{t}_{\mathrm{p}}}\right) e^{\left(\frac{-a^{\prime} t}{t_{p}}\right)}
$$

where $P_{m}$ is the initial pressure, $t_{p}$ is the time of reversal of direction of pressure, and $a^{\prime}$ is a shape parameter depending on the dimensionless scaled distance $Z$, given by

$$
Z=\frac{R}{\sqrt[3]{W}}
$$

in which $\mathrm{R}$ is the distance from the centre of a spherical shape in meters and $\mathrm{W}$ is the charge mass expressed in kilograms.

\section{Mathematical Model for Circular Plate}

We consider a circular plate subject to a uniformly distributed impulsive load $I$ of radius $r$, thickness $h$ and clamped at the edges.

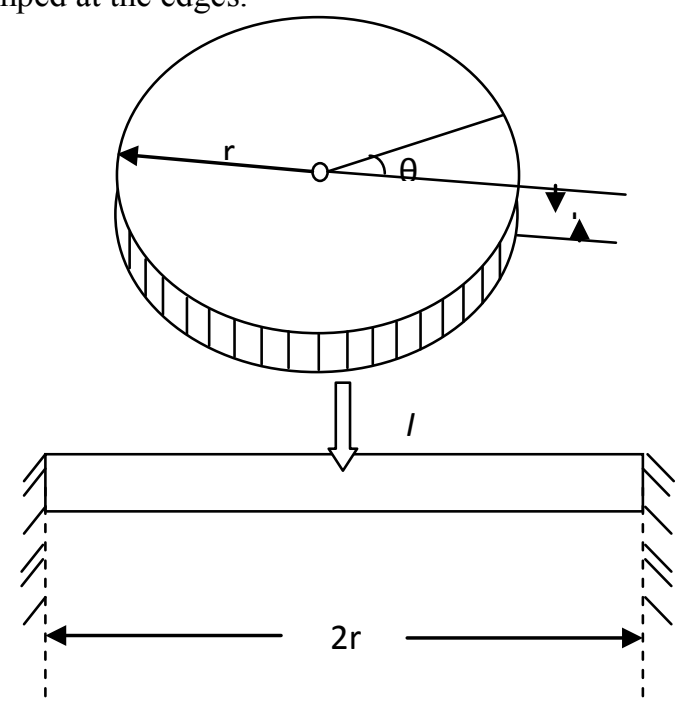

Figure 2. A schematic diagram of the clamped circular solid plate under the blast loading

\subsection{Governing Equation of Motion for a Circular Plate}

The governing equation of motion for the damped vibration of circular plate under blast loading[22] is given as

$$
[M]\left\{\ddot{a}_{n}\right\}+[C]\left\{\dot{a}_{n}\right\}+[K]\left\{a_{n}\right\}=\left\{R_{\text {ext }}\right\}
$$

where $[\mathrm{K}]$, the stiffness matrix is given as 


$$
\begin{gathered}
{[K]=\left[\begin{array}{ll}
\left(k_{M}^{p}\right)_{n m} & \left(k_{M}^{L}\right)_{n m} \\
\left(k_{M}^{L}\right)_{n m} & \left(k_{M}^{b}\right)_{n m}
\end{array}\right]} \\
(r)=-\frac{27}{2} r\left(r-\frac{1}{3}\right)(r-1) \\
N_{4}(r)=\frac{9}{2} r\left(r-\frac{1}{3}\right)\left(r-\frac{2}{3}\right)
\end{gathered}
$$

Similarly, the displacement components at any point within the element are

$$
\begin{aligned}
& {\left[\begin{array}{l}
u \\
v \\
w \\
\alpha \\
\beta
\end{array}\right]=\sum_{n=1}^{4} N_{n}\left[\mathrm{I}_{5}\right]\left[\begin{array}{l}
u_{n} \\
v_{n} \\
w_{n} \\
\alpha_{n} \\
\beta_{n}
\end{array}\right] } \\
= & \sum_{n=1}^{4} N_{n}\left[\mathrm{I}_{5}\right]\{a\}_{n}
\end{aligned}
$$

where at a node $n, u_{n}, v_{n}, w_{n}$ are the translational displacements and $\alpha_{n}$ and $\beta_{n}$ are the slop functions.

In the case of axisymmetric deformation where the integrand is independent of the tangential coordinate $\theta$ [22].

$$
\begin{aligned}
& B_{m}=\left[\begin{array}{cc}
\frac{\partial N_{m}}{\partial r} & 0 \\
\frac{1}{r} N_{m} & 0 \\
0 & -\frac{1}{r}\left(N_{m}+\frac{\partial N_{m}}{\partial r}\right)
\end{array}\right] \\
& B_{n}^{p}=\left[\begin{array}{ccc}
\frac{\partial N_{n}}{\partial r} & 0 & \frac{\partial w}{\partial r} \frac{\partial N_{n}}{\partial r} \\
\frac{1}{r} N_{n} & 0 & 0 \\
0 & -\frac{1}{r}\left(N_{n}+\frac{\partial N_{n}}{\partial r}\right) & 0
\end{array}\right] \\
& =\frac{D h}{1-v^{2}}\left[\begin{array}{cc}
\frac{\partial N n}{\partial r}\left(\frac{\partial N_{n}}{\partial r}+\frac{v}{r} N_{n}\right)+\frac{1}{r} N_{n}\left(\frac{v \partial N_{n}}{\partial r}+\frac{1}{r} N_{n}\right) & 0 \\
0 & \frac{1-v}{2 r^{2}}\left(N_{n}+\frac{\partial N_{n}}{\partial r}\right)^{2} \\
\frac{\partial w}{\partial r}\left(\frac{\partial N_{n}}{\partial r}\right)^{2}+\frac{v}{r} \frac{\partial w}{\partial r} N_{n} \frac{\partial N_{n}}{\partial r} & 0
\end{array}\right] \\
& \left(B_{n}^{S}\right)^{T}=\left[\begin{array}{cc}
\frac{\partial N_{n}}{\partial r} & 0 \\
-N_{n} & 0 \\
0 & -N_{n}
\end{array}\right] \\
& \left(B_{n}^{s}\right)^{T} D^{s} B_{n}^{s}=\frac{k D h}{2(1+v)}\left[\begin{array}{ccc}
\frac{\partial N_{n}}{\partial r} \frac{\partial N_{n}}{\partial r} & -N_{n} \frac{\partial N_{n}}{\partial r} & 0 \\
-N_{n} \frac{\partial N_{n}}{\partial r} & \left(N_{n}\right)^{2} & 0 \\
0 & 0 & \left(N_{n}\right)^{2}
\end{array}\right] \\
& \left(B_{n}^{b}\right)^{T}=\left[\begin{array}{ccc}
0 & 0 & 0 \\
\frac{\partial N_{n}}{\partial r} & \frac{1}{r} N_{n} & 0 \\
0 & 0 & \frac{\partial N_{n}}{\partial r}-\frac{N_{n}}{r}
\end{array}\right]
\end{aligned}
$$

Since the plate is clamped, we have

$$
\left(k_{M}^{L}\right)_{n m}=\int_{r}\left(B_{n}^{p}\right)^{T} D^{p} B_{m}^{l} d r=\left[\begin{array}{lll}
0 & 0 & 0 \\
0 & 0 & 0 \\
0 & 0 & 0
\end{array}\right]
$$

Applying the interpolation functions, we have the material part of the stiffness matrix to be

$$
K=\left[\begin{array}{ccc}
15220.53 v+2089.03 & 0 & 0 \\
0 & 610049.87(1-v) & 0 \\
0 & 0 & \frac{5052.99 k D h}{2(1+v)} \\
0 & 0 & -\frac{1910 k D h}{2(1+v)} \\
0 & 0 & 0
\end{array}\right.
$$

$$
\left.\begin{array}{cc}
0 & 0 \\
0 & 0 \\
-\frac{1910 k D h}{2(1+v)} & 0 \\
\frac{759.85 k D h}{2(1+v)}+\frac{h^{3}}{12\left(1-v^{2}\right)}\{241.5+1074.77 v\} & 0 \\
0 & \frac{759.85 \mathrm{kDh}}{2(1+v)}+\frac{13131.5 h^{3}}{12(1+v)}
\end{array}\right]
$$

The mass matrix $[\mathrm{M}]$ is obtain from the kinetic energy of the circular plate element

$$
\begin{aligned}
T=\frac{\rho}{2} \iint\left\{h \left[\left(\frac{\partial u}{\partial t}\right)^{2}\right.\right. & \left.+\left(\frac{\partial v}{\partial t}\right)^{2}+\left(\frac{\partial w}{\partial t}\right)^{2}\right] \\
& \left.+\frac{h^{3}}{12}\left[\left(\frac{\partial \alpha}{\partial t}\right)^{2}+\left(\frac{\partial \beta}{\partial t}\right)^{2}\right]\right\} r d r d \theta
\end{aligned}
$$

Applying the displacement components, we have

$$
T=\left[\begin{array}{c}
\dot{u}_{n} \\
\dot{v}_{n} \\
\dot{w}_{n} \\
\dot{\alpha}_{n} \\
\dot{\beta}_{n}
\end{array}\right] \iint\left[\begin{array}{ccccc}
\frac{\rho h}{2} N_{n} & 0 & 0 & 0 & 0 \\
0 & \frac{\rho h}{2} N_{n} & 0 & 0 & 0 \\
0 & 0 & \frac{\rho h}{2} N_{n} & 0 & 0 \\
0 & 0 & 0 & \frac{\rho h^{3}}{24} & 0 \\
0 & 0 & 0 & 0 & \frac{\rho h^{3}}{24}
\end{array}\right] r d r d \theta\left[\begin{array}{c}
\dot{u}_{n} \\
\dot{v}_{n} \\
\dot{w}_{n} \\
\dot{\alpha}_{n} \\
\dot{\beta}_{n}
\end{array}\right]
$$

where $[\mathrm{M}]$ the mass matrix is

$$
[M]=\iint\left[\begin{array}{ccccc}
\frac{\rho h}{2} N_{n} & 0 & 0 & 0 & 0 \\
0 & \frac{\rho h}{2} N_{n} & 0 & 0 & 0 \\
0 & 0 & \frac{\rho h}{2} N_{n} & 0 & 0 \\
0 & 0 & 0 & \frac{\rho h^{3}}{24} & 0 \\
0 & 0 & 0 & 0 & \frac{\rho h^{3}}{24}
\end{array}\right] r d r d \theta
$$

With interpolation functions and applying the axisymmetric deformation, we have

$$
[M]=\left[\begin{array}{ccccc}
\rho \mathrm{h} & 0 & 0 & 0 & 0 \\
0 & \rho \mathrm{h} & 0 & 0 & 0 \\
0 & 0 & \rho \mathrm{h} & 0 & 0 \\
0 & 0 & 0 & \frac{\rho \mathrm{h}^{3}}{12} & 0 \\
0 & 0 & 0 & 0 & \frac{\rho \mathrm{h}^{3}}{12}
\end{array}\right]
$$

The damping matrix [C] is formed as a linear combination of the stiffness and mass matrices; i.e.,

$$
[\mathrm{C}]=\alpha[\mathrm{K}]+\beta[\mathrm{M}]
$$

where $\alpha$ and $\beta$ are called the proportional damping constants.

$\left\{R_{\text {ext }}\right\}$ is the external load, in this case an exponential blast loading.

The elements equation of motion are

$$
\left[m^{(e)}\right]\left\{\ddot{a}_{n}\right\}+\left[c^{(e)}\right]\left\{\dot{a}_{n}\right\}+\left[k^{(e)}\right]\left\{a_{n}\right\}=\left\{f^{(e)}\right\}
$$

For free vibration response [10], equation (3.6) reduces to

$$
m \ddot{a}_{n}+c \dot{a}_{n}+k a_{n}=0
$$

In the case of under damped, the solution to the above equation is

$$
a_{n}(t)=e^{-y \omega t}\left(B_{1} \cos \omega_{D} t+B_{2} \sin \omega_{D} t\right)(3.7)
$$

where $w_{D}$ is the damped natural circular frequency, given by 
and,

$$
\omega_{D}^{2}=\left(1-y^{2}\right) \frac{k}{m}
$$

$$
\omega=\sqrt{\frac{k}{m}}
$$

$\mathrm{B}_{1}$ and $\mathrm{B}_{2}$ are constants to be determined using the given boundary conditions.

Solution of forced vibration

$$
[M]\left\{\ddot{a}_{n}\right\}+[C]\left\{\dot{a}_{n}\right\}+[K]\left\{a_{n}\right\}=\left\{R_{\text {ext }}\right\}
$$

Applying the transformation using the normalized matrix $\{a\}=[A]\{p\}$, equation $(3.8)$ becomes

$$
\{\ddot{P}\}+\left(\alpha+\beta\left[\omega^{2}\right]\right)\{\dot{P}\}+\left[\omega^{2}\right]\{P\}=[A]^{T}\left\{R_{\text {ext }}\right\}
$$

where $\{P\}$ is the column matrix of generalized displacements, which are linear combinations of the actual modal displacements $\{a\}$, and $[A]$ is the normalized modal matrix.

and

$[A]^{T}[M][A]=[I]$ (orthogonality principle),

$$
[A]^{T}[K][A]=\left[\begin{array}{ccccc}
\omega_{1}^{2} & 0 & 0 & 0 & 0 \\
0 & \omega_{2}^{2} & 0 & 0 & 0 \\
0 & 0 & \omega_{3}^{2} & 0 & 0 \\
0 & 0 & 0 & \omega_{4}^{2} & 0 \\
0 & 0 & 0 & 0 & \omega_{5}^{2}
\end{array}\right]=\left[\omega^{2}\right]
$$

Solving the decoupled equation

$$
\ddot{P}_{n}+\left(\alpha+\beta \omega_{n}^{2}\right) \dot{P}_{n}+\omega_{n}^{2} P_{n}=\sum_{i-1}^{5} A_{i}^{(n)} R_{i}(t)
$$

We consider one of the terms of the right hand side of equation (3.10) subject to a uniform blast described by the modified Friedlander exponential decay equation.

Equation (3.10) now becomes

$$
\ddot{p}_{n}+\left(\alpha+\beta \omega_{n}^{2}\right) \dot{p}_{n}+\omega_{n}^{2} p_{n}=P_{m}\left(1-\frac{t}{t_{p}}\right) e^{-\frac{a^{\prime} t}{t_{p}}}
$$

Equation (3.11) has the solution

$$
p_{n}(t)=p_{n h}(t)+p_{n p}(t)
$$

where the homogenous solution $p_{n h}$ is given as

$$
p_{n h}(t)=e^{-\frac{1}{2}\left(\alpha+\beta \omega_{n}^{2}\right) t}\left\{B_{3} \cos \omega_{r} t+B_{4} \sin \omega_{r} t\right\}
$$

with

$$
\omega_{r}=\sqrt{4 \omega_{n}^{2}-\left(\alpha+\beta \omega_{n}^{2}\right)^{2}}
$$

To obtain the particular integral $p_{n p}$, we then assume a solution of the form

$$
p_{n p}(t)=B_{5} e^{-\frac{a^{\prime}}{t_{p}} t}+B_{6} t e^{-\frac{a^{\prime}}{t_{p}} t}
$$

On substitution, we have

$$
\begin{aligned}
& P_{n p}(t)=\left\{\frac{P_{m} t_{p}^{2}\left(a^{\prime 2}-a^{\prime} t_{p} \alpha-a^{\prime} t_{p} \beta \omega_{n}^{2}+\omega_{n}^{2} t_{p}^{2}\right)}{\left(a^{\prime 2}-a^{\prime} t_{p} \alpha-a^{\prime} t_{p} \beta \omega_{n}^{2}+\omega_{n}^{2} t_{p}^{2}\right)^{2}}\right\} e^{-\frac{a^{\prime} t}{t_{p}}} \\
& +\frac{-t_{p}}{a^{\prime 2}-a^{\prime} t_{p} \alpha-a^{\prime} t_{p} \beta \omega_{n}^{2}+\omega_{n}^{2} t_{p}^{2}} t e^{-\frac{a^{\prime} t}{t_{p}}}
\end{aligned}
$$

Gives the general solution

$$
\begin{aligned}
& P_{n}(t) \\
& =e^{-\frac{1}{2}\left(\alpha+\beta \omega_{n}^{2}\right) t}\left\{B_{3} \cos \omega_{r} t+B_{4} \sin \omega_{r} t\right\} \\
& +\left\{\frac{-\left(2 a^{\prime} t_{p}^{2}-\alpha t_{p}^{3}+\beta t_{p}^{3} \omega_{n}^{2}\right)}{\left(a^{\prime 2}-a^{\prime} t_{p} \alpha-a^{\prime} t_{p} \beta \omega_{n}^{2}+\omega_{n}^{2} t_{p}^{2}\right)^{2}}\right\} e^{-\frac{a^{\prime} t}{t_{p}}} \\
& +\frac{-t_{p}}{a^{\prime 2}-a^{\prime} t_{p} \alpha-a^{\prime} t_{p} \beta \omega_{n}^{2}+\omega_{n}^{2} t_{p}^{2}} t e^{-\frac{a^{\prime} t}{t_{p}}}
\end{aligned}
$$

To obtain the actual displacement, we apply the transformation matrix

$$
\begin{gathered}
\left\{a_{n}\right\}=[A]\left\{P_{n}\right\} \\
\therefore\left\{a_{n}\right\}=[A]\left[e^{-\frac{1}{2}\left(\alpha+\beta \omega_{n}^{2}\right) t}\left\{B_{3} \cos \omega_{r} t+B_{4} \sin \omega_{r} t\right\}+\right. \\
P m t p 2 d 2-d^{\prime} t p \alpha-d^{\prime} t p \beta \omega n 2+\omega n 2 t p 2-2 d t p 2-\alpha t p 3+\beta t p 3 \omega \\
n 2 d^{\prime} 2-d^{\prime} t p \alpha-d^{\prime} t p \beta \omega n 2+\omega n 2 t p 22 e- \\
d^{\prime} t t p-t p d 2-d^{\prime} t p \alpha-d^{\prime} t p \beta \omega n 2+\omega n 2 t p 2 t e-d t p
\end{gathered}
$$

with the initial conditions

$$
a_{n}(0)=0, \quad \dot{a}_{n}(0)=0
$$

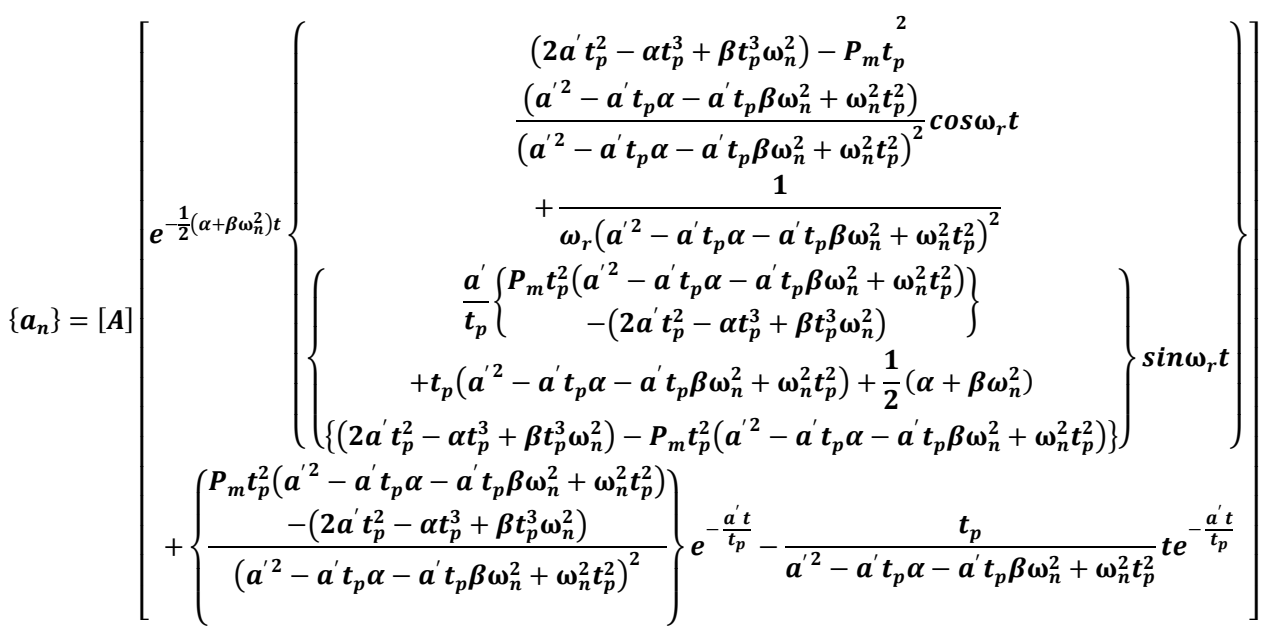




\section{Numerical Experimentation}

We employ the MAPLE 12 Mathematical Computer Software to solve the system stiffness matrix $[\mathrm{K}]$, the system mass matrix $[M]$, the fundamental frequency $\omega$, and the system force matrix $[\mathrm{P}]$. The displacement profiles of the circular plate are displayed.

The material employed is the clamped circular plate, with the following dimensions and mechanical properties.

Radius $\mathrm{r}=2 \mathrm{~m}$

Thickness $\mathrm{h}=0.003 \mathrm{~m}$

Density $\rho=2778 \mathrm{~kg} / \mathrm{m}^{3}$

Young's modulus $E=30 \times 10^{10} \mathrm{Nm}^{-2}$

Poisson's ratio $v=0.3$

The external load material properties are

$t_{p}=0.1$

$P_{m}=500 p s i$

$a^{\prime}=2$

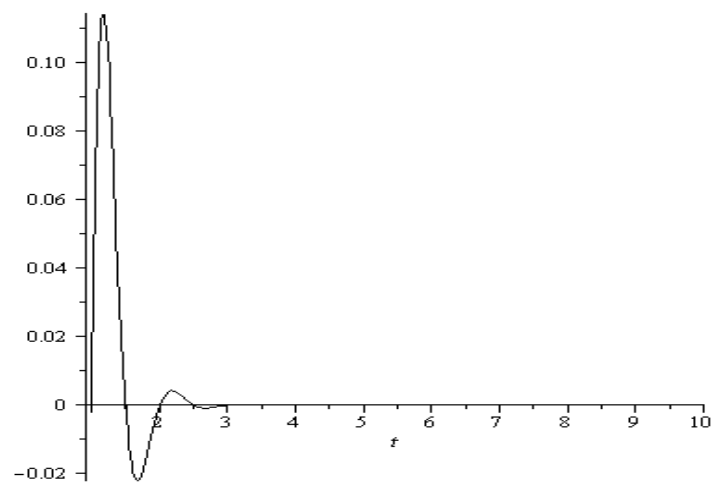

Figure 5. Vibration of Damped Circular Plate subject to Exponential Blast pulse, $\alpha=3.6, \beta=3, t_{p}=0.1$

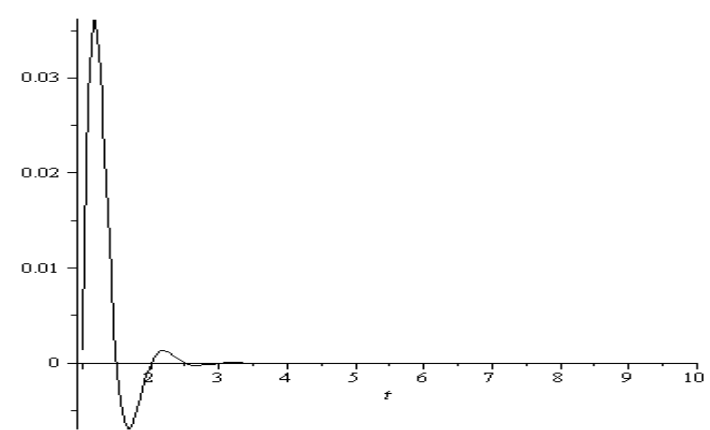

Figure 6. Vibration of Damped Circular Plate subject to Exponential Blast pulse, $\alpha=3.6, \beta=3, t_{p}=0.2$

\section{Discussion of Results}

Dynamic analyses of damped circular plate with shear deformation and rotator inertia subject to blast loading were considered. Equation (3.13) gives the dynamic response damped circular plate subjected to exponential blast loading. In fig 4.1. and fig 4.2, the effect on exponential blast loading to clamped circular plate with two different values of pulse duration $\left(t_{p}=0.1\right.$ and $\left.t_{p}=0.2\right)$ are reported. While the lowest pulse duration gives rises to the vibration amplitude, the effect on damping dies out the amplitude quickly.

\section{ACKNOWLEDGEMENTS}

We would like to sincerely and wholeheartedly thank the management and the entire staff of my institution Federal University of Technology, Minna for given us enabling environment to carry out this research work.

\section{REFERENCES}

[1] Aiyesimi, Y.M, (1999). 'The Dynamic Response of an Elastic Plate to Longitudinally Moving Concentrated Load', The Nigerian Geographical Teachers Journal Vol 2, pg 124 - 131

[2] Aiyesimi, Y.M, (1999). 'The Dynamic Analysis of an Elastic Plate with Point-Moving Load', Journal of Nigerian Association of Mathematical Physics. Vol 3,pg 249-257

[3] Aiyesimi, Y.M, (2000). 'The Analytical Investigation of the Effect of Amplitude Ratio on the Dynamic Response of a Viscoelastic Plate Subject to a cyclic Moving Load', Journal of Science, Technology \& Mathematics Education. Vol 3(2), pg 79-84

[4] Amabili, M. and Pardoussis MP (2003). 'Review of Studies on Geometrically Nonlinear Vibrations and Dynamics of Circular Cylindrical Shells and Panels, with and without Fluid - Structure Interaction', Applied Mechanics Reviews. Vol 56, No 4

[5] Chiang, C. K., Mei, C. and Gray Jr, C. E. (1991). 'Finite Element Large-Amplitude Free and Forced Vibrations of Rectangular Thin Composite Plates'. Trans. ASME, J. Vibration. Acoustics, Vol. 113, pg 309-315

[6] Chorng-Fuh Liu and Ge-Tzung Chen (1995). 'A Simple Finite Element Analysis of Axisymmetric Vibration of Annular and Circular Plates', Int. J. Mech. Sci. Vol. 37, No. 8, pg. 861-871

[7] Ding, H. Rongqiao, X. Yuwei, C. and Weiqui, C. (1999). 'Free Axisymmetric Vibration of Transversely Isotropic Piezoelectric Circular Plates', International Journal of Solids and Structures Vol. 36, pg 4629 - 4652

[8] Hutton, D. V. (2004). 'Fundamentals of Finite Element Analysis', McGraw - Hill Companies, New York

[9] Karami Khorramabadi, M., Najafizadeh, M. M., Alibabaei Shahraki, J., and Khazaeinejad, P.(2008). 'Effect of Shear Theories on Free Vibration of Functionally Graded Plates', Proceedings of World Academy of Science, Engineering and Technology. Vol 36 ISSN 2070-3740

[10] Krodkiewski, C. J. (2006). 'Mechanical Vibration', Design and print centre, University of Melboume, Lecture Note

[11] Ming-Hui Lee and Tsung-Chien Chen (2008). 'Blast Load Input Estimation of the Medium Girder Bridge using Inverse Method', Defence Science Journal, Vol. 58, No. 1, pg. 46-56

[12] Olarewaju, A. J., Kameswara Rao, N.S.V. and Mannan, M. A. (2011). 'Simulation and Verification of Blast Load Duration for Studying the Response of Underground Horizontal 
and Vertical Pipes Using Finite Element Method', EJGE Vol. Bund. G

[13] Rao, S. R., Sheikh, A. H. and Mukhopadhyay, M (1993). 'Large-Amplitude Finite Element Flexural Vibration of Plates/Stiffened Plates', Journal of the Acoustical Society of America, Vol. 93, pg 3250-3257

[14] Sadiku, S. (2006). 'Theory of Plate and Shells', Lecture Notes Unpublished, Federal University of Technology, Minna.

[15] Sastry, S. S. (1986). 'Engineering Mathematics', Prentice Hall of India, Volume Two

[16] Sathyamoorthy, M. (1996). 'Influence of Transverse Shear and Rotatory Inertia on Non-linear Vibration of Circular Plates', Computers and Structures, Vol. 60, pg 613-618

[17] Timoshenko, S. and Woinowsky-Krieger, S. (1959). 'Theory of Plates and Shells', McGraw-Hill Book Company, London, 2nd Edition
[18] Touze, C., Thomas, O. and Chaigne, A. (2002). 'Asymmetric Non-Linear Forced Vibrations of Free-Edge Circular Plates. Part 1: Theory', Journal of Sound and Vibration, Vol. 258, pg 649-676

[19] Umphai, K. D. and Mei, C. (1986). 'Finite Element Method for Non-Linear Forced Vibrations of Circular Plates', International Journal for Numerical Methods in Engineering, Vol. 23, pg 1715- 1726

[20] Van der Meer, L. J. (2008). 'Dynamic Response of High-Rise Building Structures to Blast Loading', Research report: A-2008.3, $O-2008.8$

[21] Zarowski, C. J. (2004). 'An Introduction to Numerical Analysis for Electrical and Computer Engineers', John Wiley \& Sons, Inc., New Jersey

[22] Zienkiewicz, O. C. and Taylor, R. L. (2005). 'Finite Element Method for Solid and Structural Mechanics', Butterworth Heinemann, Sixth Edition 